\title{
Whole body vibration compared to conventional physiotherapy in patients with gonarthrosis: a protocol for a randomized, controlled study
}

\author{
Gregor Stein*1,2, Peter Knoell1,2, Christoph Faymonville1, Thomas Kaulhausen¹, Jan Siewe1, Christina Otto1, Peer Eysel1
} and Kourosh Zarghooni ${ }^{1}$

\begin{abstract}
Background: Osteoarthritis $(\mathrm{OA})$ is the most common degenerative arthropathy. Load-bearing joints such as knee and hip are more often affected than spine or hands. The prevalence of gonarthrosis is generally higher than that of coxarthrosis.

Because no cure for OA exists, the main emphasis of therapy is analgesic treatment through either mobility or medication. Non-pharmacologic treatment is the first step, followed by the addition of analgesic medication, and ultimately by surgery.

The goal of non-pharmacologic and non-invasive therapy is to improve neuromuscular function, which in turn both prevents formation of and delays progression of OA. A modification of conventional physiotherapy, whole body vibration has been successfully employed for several years. Since its introduction, this therapy is in wide use at our facility not only for gonarthrosis, but also coxarthrosis and other diseases leading to muscular imbalance.

Methods/Design: This study is a randomized, therapy-controlled trial in a primary care setting at a university hospital. Patients presenting to our outpatient clinic with initial symptoms of gonarthrosis will be assessed against inclusion and exclusion criteria. After patient consent, 6 weeks of treatment will ensue. During the six weeks of treatment, patients will receive one of two treatments, conventional physiotherapy or whole-body-vibration exercises of one hour three times a week. Follow-up examinations will be performed immediately after treatment and after another 6 and 20 weeks, for a total study duration of 6 months. 20 patients will be included in each therapy group.

Outcome measurements will include objective analysis of motion and ambulation as well as examinations of balance and isokinetic force. The Western Ontario and McMaster Universities Arthritis Index and SF-12 scores, the patients' overall status, and clinical examinations of the affected joint will be carried out.
\end{abstract}

Discussion: As new physiotherapy techniques develop for the treatment of $\mathrm{OA}$, it is important to investigate the effectiveness of competing strategies. With this study, not only patient-based scores, but also objective assessments will be used to quantify patient-derived benefits of therapy.

Trial registration: Deutsches Register Klinischer Studien (DRKS) DRKS00000415

Clinicaltrials.gov NCT01037972

EudraCT 2009-017617-29

* Correspondence: gregor.stein@uk-koeln.de

1 Department of Orthopaedic and Trauma Surgery, University of Cologne, Kerpener Strasse 62, 50924 Cologne, Germany

Full list of author information is available at the end of the article

C 2010 Stein et al; licensee BioMed Central Ltd. This is an Open Access article distributed under the terms of the Creative Commons :-1Wed Central Attribution License (http://creativecommons.org/licenses/by/2.0), which permits unrestricted use, distribution, and reproduction in any medium, provided the original work is properly cited. 


\section{Background}

Osteoarthritis (OA) is the most common degenerative arthropathy. Patients affected by this disease suffer from numerous sequelae, and costs of treatment are high enough to have socio-economic relevance [1-3]. Prevalence of OA rises steadily with advancing age, and women are more typically affected than men [4-6]. Load-bearing joints in particular such as knee and hip are more often affected than spine or hands. Prevalence and incidence of clinical symptoms, and radiologically confirmed gonarthrosis range from $1.6-9.4 \%$ and 240 out of $100.000[7,8]$. The prevalence of gonarthrosis is usually higher than that of coxarthrosis, particularly in middle-aged patients [9].

Gonarthrosis is commonly defined as a degenerative, non-infectious disease of the knee joint. Morphologically, arthrosis is characterized by progressive loss of cartilage, sclerosis of the subchondral osseous structures, and partial involvement of the synovia [10].

The cardinal symptom of gonarthrosis is pain that is usually activity-related and is intensified by weight-bearing. Initially, the pain has an episodic character and can be relieved by rest, but with disease progression the pain intensifies and becomes more constant.

When patients present with severe pain, motor impairment, or joint effusion, the arthrosis is refered to as activated [9].

Further clinical signs of gonarthrosis are crepitus, palpable osteophytes, coarsening of joint contour, varus or valgus deformity, and joint instability. Because almost $40 \%$ of patients with OA are asymptomatic, Kellgren and Lawrence introduced a set of radiologic criteria in 1957. A more specific classification was developed in 1986 by the American Rheumatism Association (ARA), now called the American College of Rheumatology (ACR). These criteria have been validated and include several clinical and radiographic examinations [11]. As long as no cure exists, the main emphasis of OA treatment is analgesia, through either increasing mobility or medication administration. Initially, non-pharmacologic therapies are attempted, followed by the addition of medication, and ultimately surgical intervention [12-14].

For non-pharmacologic and non-invasive therapy, the goal of treatment is to optimize neuromuscular function, which acts both to prevent as well as to delay progression of the disease [15]. Muscle training to correct imbalance, relieve the joints, and invigorate surrounding structures is essential to this therapy. The goals of treatment are twofold--to provide analgesia for patients, but also to delay the need for pharmacotherapy. Because of this, certain enhancements to conventional physiotherapy have been introduced. One such modification is the use of whole body vibration devices, available since 2001. For this, patients stand on a vibrating plate with adjustable amplitude and frequency. The idea is to activate counter- regulatory contractions through the distension of reflexes, which leads to fine-tuning of the neuromuscular spindles. Since its introduction, this therapy has been used widely at our facility not only for gonarthrosis, but also coxarthrosis and other diseases causing muscular imbalance.

Various studies have identified a number of results after the use of whole body vibration. For instance, improvements in muscular strength and coordination as well as an arrest of muscle atrophy have been observed [16,17]. It also improves osseous metabolism [18].

The general assumption is that whole body vibration causes minor stress of the joint affected by OA. Examining women with gonarthrosis, Trans et al. found that whole body vibration led to increased muscle formation and improved proprioception [19]. Male patients have not yet been assessed.

Clearly, an investigation of the use of whole body vibration in men alone would be an interesting clinical study. However, because the success of treatment has so far been measured mostly using questionnaires, we also thought it expedient to introduce validated questionnaires like WOMAC and SF-12, and also assess balance, mobility, and general function of the lower legs. By these means, we should achieve some outlook of this technique from a long-term perspective.

\section{Objective}

The objective of this study is to compare the effects of physiotherapy alone or physiotherapy conducted with a whole body vibration device for the treatment of mild to moderate symptomatic gonarthrosis. The study would be conducted in a primary care setting at a university hospital. Questionnaire assessments relating to clinical outcome as well as objective analysis of ambulation and balance will be performed.

\section{Methods and Design}

The study is designed as a randomized, therapy-controlled trial in a primary care setting at a university hospital. Patients presenting to our outpatient clinic with earlystage gonarthrosis will be assessed against study inclusion and exclusion criteria. After patient informed consent, 6 weeks of therapy will ensue. Follow-up examinations will be performed immediately after treatment and after another 6 and 20 weeks, for a total study duration of 6 months.

Source of funding for the study is the "Deutsche Arthrose Hilfe e.V.", a non-profit patients support group promoting scientific research on osteoarthritis. Funding has been used for the conduction of therapy, sample size calculations, Womac and SF-12 licenses and for equipment acquisition. 
Experimental research in this trial will be performed with the approval of the ethics committee of the medical faculty of the University of Cologne under the reference number 10-006. Research carried out in the trial will be in compliance with the Helsinki Declaration.

\section{Participants and recruitment}

Patients aged 30 - 80 years presenting to our outpatient clinic with symptoms of early-stage gonarthrosis are eligible for the trial. Further inclusion criteria are summarized in the appendices.

Patients participating in parallel interventional studies as well as patients suffering from severe gonarthrosis are excluded from this study. The exclusion criteria are summarized in the appendices.

\section{Interventions}

During the six weeks of treatment, patients will receive one of two treatments:

- Conventional physiotherapy

Whole-body-vibration exercises

\section{Conventional physiotherapy}

Patients in this study group will attend physiotherapeutic exercise sessions of one hour three times a week for six weeks. The sessions consist of aerobic and muscle strengthening as well as coordination exercises. Patients will practice activities of daily living. The goals of these exercises are to improve joint stability, optimize knee and ankle proprioception, and advance neuromuscular innervation of the lower extremity and thereby suppress pathologic motion patterns. This should lead to optimized mobility, increased stability, and thus more endogenous analgesia of the affected joint.

\section{Whole body vibration}

Patients in this study group will attend whole body vibration exercise sessions of one hour three times a week for six weeks, using the Galileo Fitness device. Initial training sessions will focus on patient acclimatization, and afterwards improved on muscular capacity and body coordination. During exercise sessions, patients will do 6 training cycles of 3 minutes each. The goals of this treatment are improved proprioception of the ankle and knee joints, as well as optimization of neuronal reactivation of the muscles and thereby improved joint stability. This should also increase endogenous analgesia.

\section{Outcome measures and assessments}

The primary outcome measure is the patients' evaluation of improvement on the visual analogue scale of the WOMAC indices for pain and activities of daily life, comparing baseline and post-treatment. The secondary outcome measures summarized in the appendices will also be assessed at baseline and after 6,12 , and 26 weeks.
The WOMAC index is a validated patient questionnaire used to evaluate coxarthrosis and gonarthrosis by evaluating symptoms and motor impairment in daily life $[20,21]$. Use of this index in clinical studies is recommended by OARSI and EMEA and has received approval from many studies [20].

Functional motion analysis, the Modified Clinical Test of Sensory Interaction in Balance, the Tandem Walk Test, and the Rhythmic Weight Shift Test will be performed using the Leonardo Mechanography Gangway (Novotec Inc., Pforzheim, Germany) and the Balance Master Analysis System (Neurocom Inc., Clackamas, USA). These will provide reliable data on essential parts of ambulation, e.g. stride length, speed of movement, shifting of balance point, force, power, and workload.

Overall patient status is recommended as a secondary endpoint by EMEA and GREES [11,22]. According to the recommendations of Bellamy et al. [23], study participants will be asked questions regarding symptoms of the treated and other joints as well as subjective health status.

The Osteoarthritis Research Society International (OARSI) and the Outcome Measures in Rheumatology Committee (OMERACT) have developed common criteria to assess patient response to therapy for OA $[24,25]$. The criteria assess improvements of pain, affected joint function, as well as overall patient status.

The SF-12 score uses subjective patient responses to measure success of therapy and thereby evaluate quality of life. SF-12 is the abbreviated version of the SF-36 Health Survey and contains 12 items representing 8 dimensions of physical and mental fitness. In our study, the validated German translation will be used [26].

The clinical examination of the knee joint will assess intraarticular effusion as well as range of motion.

Finally, the isokinetic force of the knee will be measured using a calibrated "Biodex System 3" (Biodex Medical Systems Inc., Shirley, NY, USA). The maximum isometric force will be measured first by extending and then by flexing the knee.

\section{Sample size}

It is assumed that the standard deviation of the therapeutic effects is $\sigma=19 \mathrm{~mm}$. Furthermore, no difference between the effects of the different therapy groups is expected. The relevant difference in the scores used for primary outcome is defined as $20 \mathrm{~mm}$. By postulating $80 \%$ power using a level of significance of $2.5 \%$, a total number of 31 test patients is needed. When considering a potential drop-out of 4 patients per treatment group, a total of 40 patients will be included into the study.

\section{Randomization}

The randomization of patients into the groups of intervention and control is achieved by using blocks of ran- 
domized size. The groups itself are furthermore stratified into gender groups. Technically a locked container with sequentially numbered envelopes is used. The random allocation sequence was generated by the institute of medical statistics, informatics and epidemiology of the university conducting the trial. Enrollment and randomization will be executed by the investigator.

\section{Discussion}

OA remains a major disease in the field of musculoskeletal disorders. Patients presenting with early-stage OA are typically treated with non-curative therapies such as analgesics. Until a cure is found, therapy focused on the roots of symptomatic complaints should be used as well to bridge the gap between disease onset and operative therapy such as partial or total joint replacement. Conventional physiotherapy is well established as one alternative for this bridge. However, new treatment methods like whole body vibration training also seek to improve muscle coordination and thereby optimize affected joint loading. Because both conventional physiotherapy and whole body vibration have co-existed for several years, we can now compare them in an attempt to optimize non-operative treatment for patients with OA.

\section{Appendix}

Inclusion criteria:

- Age 30 - 80 years

- Body weight less than or equal to $160 \mathrm{~kg}$

- Body-Mass-Index less than $40 \mathrm{~kg} / \mathrm{m}^{2}$

- Outpatient

- Legal competence

- Signed informed consent

- Uni- or bilateral gonarthrosis according to ACR criteria

- WOMAC-pain index (visual analogue scale) of 30-

$70 \mathrm{~mm}$

- Gonarthrosis stage II-III according to Kellgren and Lawrence

Exlusion criteria:

- Participation in parallel interventional studies

- Bilateral gonarthrosis with WOMAC Pain index $\geq$ $70 \mathrm{~mm}$

- Dominant femoro-patellar gonarthrosis

- Previous surgery during the past 6 months (exception: meniscus surgery: past 3 months)

- Injury of the study joint during the last 6 months - Secondary rheumatoid or septic arthrosis or systemic diseases affecting the study joint

- Activated gonarthrosis with intraarticular effusion

- Body weight $>160 \mathrm{~kg}$ or body mass index $>40 \mathrm{~kg} / \mathrm{m}^{2}$

- Analgesic therapy with steroidal drugs

- Physiotherapy of the lower extremities during the past 6 weeks
- Existing endoprothesis in the lower extremities

Secondary outcome measures:

- Functional motion analysis (Walk Across Test)

- Modified Clinical Test of Sensory Interaction in Bal-

ance

- Tandem Walk Test

- Rhythmic Weight Shift Test

- WOMAC global index

- Overall patient status

- Response using the criteria of OMERACT-OARSI

- Quality of life measured by SF- $12^{\circ}$

- Clinical examination of the knee joint

- Isokinetic measurement of forces in the knee joint

\section{Competing interests}

The authors declare that they have no conflicting or competing interests in carrying out this study. Deutsche Arthrose-Hilfe e.V., a non-profit patient support organization, will support the study. None of the authors receives or has received any funding from the Deutsche Arthrose-Hilfe e.V. Deutsche ArthroseHilfe e.V. has not been involved in the design of the study and will not be involved in analysis of the data and publications.

\section{Authors' contributions}

GS and KZ prepared the study and participated in its design and conduction. GS conceived of the study. All authors revised the manuscript and participated in the conduction of the study. All authors read and approved the final manuscript.

\section{Acknowledgements}

Hans-Philipp Springorum contributed in the acquisition of funding and general revision of the manuscript. Peer Eysel generally supervises the research group and provides general support.

Source of funding for the study is the "Deutsche Arthrose Hilfe e.V.", a non-profit patients support group promoting scientific research on osteoarthritis.

\section{Author Details}

'Department of Orthopaedic and Trauma Surgery, University of Cologne, Kerpener Strasse 62, 50924 Cologne, Germany and ${ }^{2}$ Clinical trials centre, BMBF 01 KN0706, University of Cologne, Kerpener Strasse 62, 50924 Cologne, Germany

Received: 14 April 2010 Accepted: 21 June 2010

Published: 21 June 2010

\section{References}

1. Adams ME, Atkinson MH, Lussier AJ, Schulz Jl, Siminovitch KA, Wade JP, Zummer M: The role of viscosupplementation with hylan G-F 20 (Synvisc) in the treatment of osteoarthritis of the knee: a Canadian multicenter trial comparing hylan G-F 20 alone, hylan G-F 20 with nonsteroidal anti-inflammatory drugs (NSAIDs) and NSAIDs alone. Osteoarthritis Cartilage 1995, 3(4):213-25.

2. Pelletier JP, Martel-Pelletier J: The pathophysiology of osteoarthritis and the implication of the use of hyaluronan and hylan as therapeutic agents in viscosupplementation. J Rheumatol Supp/ 1993, 39:19-24.

3. Wobig M, Bach G, Beks P, Dickhut A, Runzheimer J, Schwieger G, Vetter G, Balazs E: The role of elastoviscosity in the efficacy of viscosupplementation for osteoarthritis of the knee: a comparison of hylan G-F 20 and a lower-molecular-weight hyaluronan. Clin Ther 1999, 21(9):1549-62.

4. Cunningham LS, Kelsey JL: Epidemiology of musculoskeletal impairments and associated disability. Am J Public Health 1984, 74(6):574-9.

5. Mikkelsen WM, Dodge HJ, Duff IF, Kato H: Estimates of the prevalence of rheumatic diseases in the population of Tecumseh, Michigan, 1959-60. J Chronic Dis 1967, 20(6):351-69.

6. van Saase $J L$, van Romunde LK, Cats A, Vandenbroucke JP, Valkenburg HA: Epidemiology of osteoarthritis: Zoetermeer survey. Comparison of 
radiological osteoarthritis in a Dutch population with that in 10 other populations. Ann Rheum Dis 1989, 48(4):271-80.

7. Felson DT, Zhang Y: An update on the epidemiology of knee and hip osteoarthritis with a view to prevention. Arthritis Rheum 1998, 41(8):1343-55.

8. Oliveria SA, Felson DT, Reed Jl, Cirillo PA, Walker AM: Incidence of symptomatic hand, hip, and knee osteoarthritis among patients in a health maintenance organization. Arthritis Rheum 1995, 38(8):1134-41.

9. Duchow J, Kohn D: Treatment of knee joint arthritis in the middle-aged patient. Orthopade 2003, 32(10):920-29. quiz 30

10. Pullig O, Pfander D, Swoboda B: Molecular principles of induction and progression of arthrosis. Orthopade 2001, 30(11):825-33.

11. Altman R, Asch E, Bloch D, Bole G, Borenstein D, Brandt K, Christy W, Cooke TD, Greenwald R, Hochberg M: Development of criteria for the classification and reporting of osteoarthritis. Classification of osteoarthritis of the knee. Diagnostic and Therapeutic Criteria Committee of the American Rheumatism Association. Arthritis Rheum 1986, 29(8):1039-49.

12. ACR: Recommendations for the medical management of osteoarthritis of the hip and knee: 2000 update. Arthritis Rheum 2000, 43(9):1905-15. American College of Rheumatology Subcommittee on Osteoarthritis Guidelines

13. Hochberg MC, Altman RD, Brandt KD, Clark BM, Dieppe PA, Griffin MR, Moskowitz RW, Schnitzer TJ: Guidelines for the medical management of osteoarthritis. Part II. Osteoarthritis of the knee.American College of Rheumatology. Arthritis Rheum 1995, 38(11):1541-6.

14. Steinmeyer J: Drug therapy of arthrosis. Orthopade 2001, 30(11):856-65.

15. Sharma $L$ : The role of proprioceptive deficits, ligamentous laxity, and malalignment in development and progression of knee osteoarthritis. J Rheumatol Suppl 2004, 70:87-92.

16. Da Silva-Grigoletto ME, Vaamonde DM, Castillo E, Poblador MS, GarciaManso JM, Lancho JL: Acute and cumulative effects of different times of recovery from whole body vibration exposure on muscle performance. J Strength Cond Res 2009, 23(7):2073-82.

17. Rees SS, Murphy AJ, Watsford ML: Effects of whole-body vibration exercise on lower-extremity muscle strength and power in an older population: a randomized clinical trial. Phys Ther 2008, 88(4):462-70

18. Rauch F: Vibration therapy. Dev Med Child Neurol 2009, 51(Suppl 4):166-8.

19. Trans T, Aaboe J, Henriksen M, Christensen R, Bliddal H, Lund H: Effect of whole body vibration exercise on muscle strength and proprioception in females with knee osteoarthritis. Knee 2009, 16(4):256-61.

20. Bellamy N: WOMAC: a 20-year experiential review of a patient-centered self-reported health status questionnaire. J Rheumatol 2002, 29(12):2473-6.

21. Bellamy N, Buchanan WW, Goldsmith CH, Campbell J, Stitt LW: Validation study of WOMAC: a health status instrument for measuring clinically important patient relevant outcomes to antirheumatic drug therapy in patients with osteoarthritis of the hip or knee. J Rheumatol 1988, 15(12): $1833-40$

22. EMEA: Committee for proprietary medicinal products (CPMP). Points to consider on clinical ionvestigations of medicinal products used in the treatment of Osteoarthritis (CPMP/EWP/784/97). Electronic Citation 1998.

23. Bellamy N: The WOMAC Knee and Hip Osteoarthritis Indices: development, validation, globalization and influence on the development of the AUSCAN Hand Osteoarthritis Indices. Clin Exp Rheumatol 2005, 23(5 Suppl 39):S148-53

24. Bellamy N, Campbell J, Robinson V, Gee T, Bourne R, Wells G: Viscosupplementation for the treatment of osteoarthritis of the knee. Cochrane Database Syst Rev 2005:CD005321.

25. Pham T, van der Heijde D, Altman RD, Anderson JJ, Bellamy N, Hochberg M, Simon L, Strand V, Woodworth T, Dougados M: OMERACT-OARSI initiative: Osteoarthritis Research Society International set of responder criteria for osteoarthritis clinical trials revisited. Osteoarthritis Cartilage 2004, 12(5):389-99.

26. Radoschewski M, Bellach BM: The SF-36 in the Federal Health Survey-possibilities and requirements for application at the population level. Gesundheitswesen 1999. 61 Spec No:S191-9

Pre-publication history

The pre-publication history for this paper can be accessed here: http://www.biomedcentral.com/1471-2474/11/128/prepub

doi: $10.1186 / 1471-2474-11-128$

Cite this article as: Stein et al., Whole body vibration compared to conventional physiotherapy in patients with gonarthrosis: a protocol for a randomized, controlled study BMC Musculoskeletal Disorders 2010, 11:128

\section{Submit your next manuscript to BioMed Central and take full advantage of:}

- Convenient online submission

- Thorough peer review

- No space constraints or color figure charges

- Immediate publication on acceptance

- Inclusion in PubMed, CAS, Scopus and Google Scholar

- Research which is freely available for redistribution
C Biomed Central 